DIAGNOSTICS OF PARTICLE GENESIS AND GROWTH IN RF SILANE PLASMAS BY ION MASS SPECTROMETRY AND LIGHT SCATTERING

\author{
Ch. Hollenstein, J.-L. Dorier, J. Dutta \& \\ A.A. Howling \\ submitted to Plasma Source Science and \\ Technology
}

Presented at NATO Advanced Research Workshop

"Formation, Transport and Consequences of Particles in Plasmas" Château de Bonas, France, August 30 - September 3, 1993 


\title{
Diagnostics of Particle Genesis and Growth in RF Silane Plasmas by Ion Mass Spectrometry and Light Scattering
}

\author{
Ch. Hollenstein, J.-L. Dorier, J. Dutta, L. Sansonnens and A. A. Howling \\ Centre de Recherches en Physique des Plasmas, \\ Ecole Polytechnique Fédérale de Lausanne, \\ Av. des Bains 21, CH-1007 Lausanne, Switzerland
}

Short Title: Diagnostics of Particle Genesis and Growth...

PACS numbers : $52.80 . \mathrm{Hc}, 52.80 . \mathrm{Pi}, 82.80 . \mathrm{Ms}, 82.40 . \mathrm{Ra}$

\begin{abstract}
Experimental identification of the precursors and processes leading to particles is essential for understanding particulate contamination in deposition plasmas. We have investigated particle formation in rf silane plasmas using light scattering (elastic and inelastic) and quadrupole ion mass spectrometry as complementary plasma diagnostics. Negative ions reach high masses (at least $500 \mathrm{amu}$ ) and are the only elementary species with a residence time on the scale of the powder formation time. Furthermore, a negative ion polymerisation scheme shows that the densities of high mass anions are strongly diminished at $\mathrm{kHz}$ power modulation frequencies, where reduced powder production is also observed. We conclude that negative ions are the particle precursors and that initial clusters grow by negative ion polymerisation in silane plasmas. In situ light scattering techniques are described to self-consistently determine particle size, number density and refractive index. Novel, visible photoluminescence measurements from particles suspended in the plasma are also reported. These diagnostics demonstrate that particle evolution proceeds by an agglomeration phase and that the particle properties are different from the bulk material early in the particle development.
\end{abstract}




\section{INTRODUCTION}

Particulate contamination formed in plasmas for industrial applications presents a major problem by limiting manufacturing productivity and device reliability [1-3]. The suppression of particle formation in silane plasmas for amorphous silicon deposition presupposes a knowledge of the powder precursor identity and of the reactions leading from the monosilicon process gas to particles containing millions of silicon atoms. In this paper we simultaneously use quadrupole ion mass spectrometry and light scattering to investigate precursor formation and particle growth in silane if plasmas.

Various species could be involved in plasma polymerisation leading to particle precursor formation. Polymerisation could propagate by insertion of silane radicals into higher saturated molecules $[4,5]$ or by silane addition to positive ions $[4,6]$. Negative ions are trapped in the plasma by the sheath potentials which suggests that plasma polymerisation could also proceed via negative ion pathways even if the reaction rates are slow [7-12]. Time-resolved mass spectrometric measurements of the diffusion of heavy polymerised negative ions from powermodulated if silane plasmas are modelled by a simple polymerisation scheme to demonstrate the effect of modulation on precursor formation.

Particle precursors are too small to be visible, although particles suspended in the plasma can be detected by light scattering [1-3,13-19]. The scattering is often monitored simply as a guide to the amount of powder and onset rate [20] because the intensity is a complex convolution of particle size, number density and refractive index [21,22]. Different methods have been applied to deconvolve the particle parameters using angular dissymmetry [23] and polarisation-sensitive diagnostics. In general, additional ex situ measurements [15] or assumptions (such as using the refractive index of amorphous silicon [18]) are necessary. In this paper, we attempt to selfconsistently determine the particle size, number density and refractive index from $90^{\circ}$ polarisation-sensitive scattering and extinction measurements. In addition, visible photoluminescence from the suspended particles [24] is briefly described and proposed as a potential in situ diagnostic. 


\section{EXPERIMENTAL DESCRIPTION}

The conventional parallel-plate if reactor comprises two symmetric $130 \mathrm{~mm}$ diameter electrodes with a $25 \mathrm{~mm}$ electrode gap, described in detail elsewhere [25]. Plasma parameters for good quality a-Si:H deposition were chosen: $30 \mathrm{sccm}$ pure silane flow at $0.1 \mathrm{mbar}$ pressure. The rf power of 4 to $10 \mathrm{~W}$ at an excitation frequency of $30 \mathrm{MHz}$ was capacitively-coupled to the $\mathrm{ff}$ electrode.

\section{A. Experimental Method for Light Scattering Measurements}

Light scattering by a spherical, homogeneous particle is described by the Lorentz-Mie theory [21,22]. For a randomly-distributed ensemble the total scattered intensity is the sum of the individual particle intensities provided that no multiple scattering occurs. For identical particles, the power at the detector is :

$$
\mathrm{W}_{\mathrm{s}}^{\perp} \propto \mathrm{N}_{\mathrm{p}} \Delta \mathrm{V} \Delta \Omega\left|\mathrm{S}_{\perp}\right|^{2} \mathrm{I}_{\mathrm{o}}^{\perp}
$$

and equivalently for $\mathrm{W}_{\mathrm{s}} / 1$, where the polarisations are represented in Figure 1. The terms $S_{\perp}, S_{/ /}$ are complex elements of the amplitude scattering matrix [26] and are a function of the complex refractive index $\underline{n}$, the size parameter $x=2 \pi r / \lambda(r$ is the particle radius and $\lambda$ the source wavelength) and the scattering angle $\theta\left(90^{\circ}\right.$ in our case). $N_{p}$ is the number density of particles present in the observation volume $\Delta \mathrm{V}$. Note that $\Delta \mathrm{V}$ and the solid angle $\Delta \Omega$ must be sufficiently small for the scattering angle to be close to $90^{\circ}$ and for the purity of polarisation to be conserved. The incident intensities $\left(\mathrm{I}_{0}{ }^{\perp}, \mathrm{I}_{\mathrm{O}} / /\right)$ at the observation volume are corrected for any absorption and scattering losses from powder between the source and this volume. The experiment is also arranged to avoid scattering and absorption losses between the observed volume and the detector. The measured transmitted power fraction $\mathrm{W}_{t} / \mathrm{W}_{\mathrm{o}}$ is independent of polarisation and, for simple scattering, is given by :

$$
\mathrm{W}_{\mathrm{t}} / \mathrm{W}_{\mathrm{O}}=\exp \left(-\mathrm{C}_{\text {ext }} \mathrm{N}_{\mathrm{p}} \mathrm{d}\right)
$$

where $d$ is the path length of the visible beam in the powder layer suspended in the plasma. $C_{e x t}$ is the extinction cross-section (sum of scattering and absorption) and depends on $\mathrm{x}$ and $\underline{\mathrm{n}}$. 
Experimental procedure The scattered power is monitored during particle growth with both the source and detector polarised parallel or perpendicularly, giving $W_{s} \perp$ and $W_{s}{ }_{s}$ in consecutive experiments. Since we arrange for $\mathrm{I}_{0}^{\perp}=\mathrm{I}_{0} /$, we can define :

$$
\mathrm{P}=\frac{\mathrm{W}_{\mathrm{s}}^{\perp}-\mathrm{W}_{\mathrm{S}}^{\prime \prime}}{\mathrm{W}_{\mathrm{S}}^{\perp}+\mathrm{W}_{\mathrm{S}}^{\prime \prime}}=\frac{\left|\mathrm{S}_{\perp}\right|^{2}-\left|\mathrm{S}_{\prime \prime}\right|^{2}}{\left|\mathrm{~S}_{\perp}\right|^{2}+\left|S_{\|}\right|^{2}}
$$

which would be equivalent to the degree of linear polarisation for the case of unpolarised incident light. Note that $P$ is a function of $x$ and $\underline{n}$ only, and $S_{\perp}$ and $S_{/ /}$, which also determine $C_{\text {exl }}$, are calculated using the BHMIE algorithms [26]. The signal evolution has been demonstrated to be highly reproducible even down to the smallest features. $\mathrm{P}$ is a multi-valued function of the size parameter and therefore an estimation of particle size requires that the particle development be followed from the beginning. The technique therefore cannot be used for instantaneous measurements in an already-formed plasma/particle system.

We find that there is no unique refractive index which can describe the time variation of $P$ assuming particle growth with continuous, monotonically-increasing radii. Therefore we must allow for a changing refractive index $\underline{n}(\mathrm{t})$ during particle growth, and follow an iterative procedure to self-consistently determine the parameters $\left\{\mathrm{N}_{\mathrm{p}}, \mathrm{x}, \underline{\mathrm{n}}\right\}$ as a function of time:

i) Firstly, the $P$ curve is reproduced using $S_{\perp}(t)$ and $S_{/ /}(t)$ values calculated from an assumed step by step variation for the real and complex parts of $\underline{n}(\mathrm{t})$ during the particle growth phase, along with the corresponding size parameter variation $x(t)$.

ii) The value of $C_{\text {ext }}(t)$ is then calculated using these $S_{\perp}$ and $S_{/ /}$values, and the timevarying particle number density $\mathrm{N}_{\mathrm{p}}(\mathrm{t})$ is estimated from the $\mathrm{W}_{\mathrm{t}} / \mathrm{W}_{\mathrm{o}}$ measurement and Eq.(2).

iii) Using Eq.(1), values for $\mathrm{W}_{\mathrm{s}} \perp$ and $\mathrm{W}_{\mathrm{s}}$ " are calculated and compared with the experimentally-measured $\mathrm{W}_{\mathbf{s}}^{\perp}$ and $\mathrm{W}_{\mathbf{s}}$ "I signals.

The procedure is iterated until a reasonably-accurate self-consistent agreement is found for the measured values of $\left\{W_{s} \perp, W_{s}^{\prime \prime}, W_{t}\right\}$ in terms of the particle parameters $\left\{\underline{n}, x, N_{p}\right\}$ over the whole time period investigated.

Limitations of the technique. The method described is non-perturbative, easy to install in a plasma reactor, and gives self-consistent results without requiring any ex-situ measurements. However, to follow the particle evolution, it is necessary to arrange for the observation volume to coincide with a position in the discharge region where the particles form and grow without drifting out of 
the zone under observation. Displacement of the particle cloud can be minimised by using an isothermal environment to eliminate thermophoretic forces [27] and by using conditions which avoid rapid production of high particle densities leading to instabilities which can empty particles from the plasma - light scattering diagnostics could wrongly interpret these effects as a real reduction in particle formation rates.

Boufendi et al [15] have demonstrated that particles are mono-sized during the early stage of development. Moreover, since the scattering cross-section varies as $\mathrm{r}^{6}$ for the smallest particles (Rayleigh scattering for $\mathrm{x}<0.1-0.3$, depending on the refractive index [28]) and at least as $\mathrm{r}^{2}$ for larger particles, our assumption of identical particles is justifiable by considering that light scattering from the initial class of particles will dominate the detected signal during the particle development investigated here.

The iterative data interpretation procedure is not automatic, but requires a degree of judgement and experience to aid in identifying the parameter trends most likely to yield selfconsistent results.

\section{B. Ion Mass Spectrometry}

The differentially-pumped mass spectrometer was a Hiden Analytical Limited Plasma Monitor type HAL-EQP 500 ${ }^{\dagger}$ for masses 1-500 amu. The ion extractor head in Figure 1 was positioned with its axis in the electrode plane $10 \mathrm{~mm}$ beyond the grounded guard screen [12]. In this work, therefore, ions were not extracted at the grounded electrode but at the mass spectrometer head which was at the same potential. Ions enter the monitor through a $100 \mu \mathrm{m}$ aperture in the extractor electrode. A $+40 \mathrm{~V}$ bias gave a good negative ion signal without creating a visible secondary plasma in the extraction orifice. For time-resolved measurements, the channeltron pulses triggered by ion events were registered by a gated counter [12]. The deposited film or powder can block the aperture [8] and the monitor extraction head had to be dismantled and cleaned in acid after every few hours of exposure to the plasma.

\section{RESULTS}

$\dagger$ Hiden Analytical Limited, Gemini Business Park, Warrington WA5 5TN, UK 


\section{A. Negative ions}

Figure 2 shows the time-resolved behaviour of mono- through to hexa-silicon hydride anions in an on/off power-modulated plasma [12]. The double peak in the mono-, di- and trisilicon hydride anion groups is predicted by the model of Overzet et al [29] : the first peak is due to attachment in the decaying sheath after plasma extinction and the second peak is due to negative ions diffusing from the plasma bulk when the sheath has collapsed [11,12,30,31]. Fig. 2 also shows that only the monosilicon hydride anions have enough time to completely disappear at 1 $\mathrm{kHz}$ modulation frequency. Because of the mass-dependent diffusion loss rates [12] the modulation frequency influences the composition of the plasma. This point will be demonstrated by a simple polymerisation model below.

Negative polysilicon hydride ionic clusters have been observed up to the $500 \mathrm{amu}$ mass limit of the mass spectrometer [32], corresponding to $\left[\mathrm{Si}_{16} \mathrm{H}_{\mathrm{x}}\right]^{-}$, indicating that negative ions could be precursors to larger clusters and ultimately to powder particles. These negative molecular ions are singly-charged by incorporation of an electron into a molecular orbital; the concept of sheaths and floating potential only apply when the object is large enough so that it is statistically meaningful to speak of electron and positive ion fluxes to its surface $[9,33,34]$. The positive ion masses are limited to only $150 \mathrm{amu}$, possibly because of the activation energy barriers described by Mandich et al [35]. The positive ion size might also be limited because of ion-ion recombination losses, or because they are efficiently evacuated from the plasma across the sheaths.

\section{Polymerisation scheme for negative ions}

Experimental results suggest that a polymerisation pathway proceeds via negative ion clustering; they are not simply by-products formed by electron attachment to a neutral species pathway. Three reasons why the high-mass negative ions are not formed by attachment to heavy neutrals are [12]:

i) no neutrals with masses as high as negative ions are detected in the mass spectra,

ii) negative ions and neutrals have very different abundance ratios,

iii) no attachment peaks exist in the time-resolved measurements for negative ions heavier than trisilicon hydride anions (Fig. 2). 
One possible polymerisation pathway which propagates via negative ions is by silane addition [7], analogous to the condensation reactions proposed for positive ions $[4,6,36]$. Ion-ion recombination is the second possible pathway for polymerisation [37,38]. Since heavy neutrals are not observed, it must be supposed that they are re-attached and therefore ion-molecule and ion-ion reactions both eventually lead to stable higher-mass negative ions. The conditions for negative ion formation are discussed in more detail in Ref. 12.

The qualitative behaviour of negative ion polymerisation in modulated silane plasmas can be described by a phenomenological model [12]. In the following, the term $S_{n}(t)$ designates the time-varying plasma density of all the polysilicon hydride anions containing $\mathbf{n}$ silicon atoms. The conservation equation for the monosilicon hydride anion density is :

monomer : $\quad \mathrm{dS}_{1}(\mathrm{t}) / \mathrm{dt}=\mathrm{f}(\mathrm{t})-\mathrm{K}_{1} \mathrm{~S}_{1}(\mathrm{t})-\mathrm{g}_{1}(\mathrm{t})$,

where $f(t)$ is the monomer source term due to electron attachment on silane, $K_{1}$ is the reaction rate for silane addition, and $\mathrm{g}_{1}(\mathrm{t})$ is the monomer loss rate due to detachment and modulation losses. For simplification of the model, we will arbitrarily assume that polymerisation proceeds by ionmolecule reactions and neglect ion-ion recombination. For the remaining equations we ignore formation of higher-mass negative ions $S_{n}(n>1)$ by any process other than by polymerisation from the preceding $S_{n-1}$ ion, to obtain :

$$
\text { polysilicon hydride anions : } \quad d_{n}(t) / d t=K_{n-1} S_{n-1}(t)-K_{n} S_{n}(t)-g_{n}(t) .
$$

The function $g_{n}(t)$ represents modulation-induced loss rates due to the sheath collapse which are considered to be large compared to the weak transverse diffusion losses out from between the electrodes. Time-averaging over a power modulation cycle in steady-state conditions and adding the first $\mathrm{N}$ equations gives :

$$
\text { mean polysilicon hydride anion density : } \quad \overline{S_{N}}=\left(\bar{f}-\sum_{1}^{N} \overline{g_{n}}\right) / K_{N}
$$

where it is assumed that there is no break in the polymerisation sequence since all $\mathrm{K}_{\mathrm{n}}$ are taken to be non-zero. Equation (6) shows that the time-averaged plasma density of the polymerised negative ion $S_{N}$ is determined by the monosilicon hydride anion production rate, the polymerisation reaction rate $\mathrm{K}_{\mathrm{N}}$ for the ion itself, and the total of the modulation-induced losses of all the preceding negative ions. This emphasises the importance of power modulation for 
controlling the density of negative, highly-polymerised species in the plasma. We estimate the time window for anion loss during the afterglow by extrapolation from experimental data as a function of modulation frequency and anion mass [12]. Steady-state time-averaged negative ion densities for a given modulation frequency are determined by iterating equations (4) and (5) until the time-variation of each ion density was the same in successive cycles. Values for $g_{n}$ and $K_{n}$ were self-consistently chosen so that the calculated losses gave a reasonable fit to the experimental data [12].

Figure 3(a) and (b) show how the time-averaged negative and positive ion signals vary with modulation frequency. Above $15 \mathrm{kHz}$ the sheath does not have time to collapse sufficiently for any negative ions to escape. Figure 3(c) shows the modelled losses for comparison with Fig. 3(b), and the corresponding calculated anion densities are shown in Fig. 3(d). The time-averaged negative ion densities in the limits of high and low modulation frequency are equal and half of the continuous plasma values for a $50 \%$ duty cycle [12]. In the intermediate frequency range around $1 \mathrm{kHz}$ the densities decrease strongly for the higher masses. The implications for powder formation are discussed in Section IV.

\section{B. Light scattering results}

Figures 4(a) and (b) show the measured signals with the fitted points from the iteration procedure superposed. Figure 4(c) shows the concomitant particle radius, number density and refractive index. We can distinguish several stages of particle formation:

For the first 35 seconds after plasma ignition, no scattered signal is observable. The particle precursors and nucleation centres are below the detection limit.

From 35 to 50 seconds, only the perpendicularly-polarised light is measurable, corresponding to Rayleigh scattering - the particle radius is less than $15 \mathrm{~nm}$.

From 50 to 80 seconds, the fitting procedure gives a reasonable fit to the raw data (see Fig. 4(a)) for which the corresponding $\{\underline{n}, x, N p\}$ values are shown in Fig. 4(c).

At least three different refractive index values are necessary to self-consistently describe the whole particle evolution. An initial rapid increase in particle radius is accompanied by a strong decrease in number density (50 to $53 \mathrm{~s}$ ), and could be interpreted as an agglomeration of invisibly-small particle precursors. These particles show a high refractive index [39]. A particle 
growth period ensues with a moderate increase in radius at almost constant number density. This general trend of an early agglomeration phase followed by slow particle growth reproduces the observations of Bouchoule et al [40], and the final number density is comparable to other work in silane-containing plasmas $[15,18]$.

As the particles grow further, the refractive index tends towards that of amorphous silicon bulk material. At $76 \mathrm{~s}$, there is an additional change where the radii increase strongly concurrently with a fall in number density. The volume fraction of powder, defined as $N_{p} 4 \pi r^{3} / 3$, remains approximately constant up to this time and so this appears to be a second phase of particle aggregation. This is supported by Transmission Electron Microscopy which shows both single smooth spheres and aggregates of spheres; the latter having the resemblance of a blackberry. During this aggregation, the measured cross-polarisation intensity $\mathrm{W}_{\mathbf{s}}{ }^{\perp /}$ becomes non-negligible. Assuming that multiple scattering can be neglected, this indicates that the particles are no longer purely spherical [41]; the fit is less accurate in this region. Finally, the scattered intensities for both polarisations fall sharply, coinciding with a rise in transmitted intensity and accompanied by strong fluctuations in plasma light emission. This is due to instabilities of the plasma/powder ensemble which eject powder from the observation volume.

Photoluminescence Measurements were carried out for the first time on particles suspended in the plasma by illuminating with a $488 \mathrm{~nm}$ argon ion laser and monitoring the spectrum at $90^{\circ}$ via a $530 \mathrm{~nm}$ high-pass filter and optical multichannel analyser [24] with an arrangement similar to Fig. 1. The visible photoluminescence in Figure 5 is suggested to originate from quantum confinement effects in nano-crystallites [24] and as such is a probe for the particulate structure. The spectral shift with time as shown in the figure reveals an evolution in the particle morphology during its formation.

\section{Light Scattering in Power-Modulated Plasmas}

Figure 6 shows the powder onset rate (defined as the inverse of the powder appearance time) and the volume of powder material in the horizontal layer at the position of the laser beam (estimated from the product of volume fraction and layer volume), for different modulation frequencies. 
Powder formation is strongly reduced for modulation frequencies below $1 \mathrm{kHz}$, in agreement with other work $[8,16-19]$.

The time development of ionic species and scattered light intensity are compared in Figure 7(a) for a square-wave power-modulated plasma (50\% duty cycle) at $2 \mathrm{kHz}$ modulation frequency. Both positive and negative ion fluxes vary on a slow timescale similar to the powder appearance time of $16 \mathrm{~s}$ before reaching steady-state levels. Comparison with Fig. 7(b), for a 10 $\%$ duty cycle in otherwise identical plasma conditions, shows that the negative ion flux time dependence is markedly different in the absence of powder formation although the positive ion flux behaviour is relatively unchanged. Figure 8 represents the steady-state ion fluxes, the total scattered intensity and the powder onset rate for a range of duty cycles at $2 \mathrm{kHz}$ modulation frequency. The negative ion loss flux is again anti-correlated with the scattered light intensity and powder onset rate, all of which vary strongly compared to the positive ion flux.

\section{DISCUSSION}

Particles large enough to be visible by light scattering form in the rf silane plasma only after many seconds as shown in Figs. 4 and 7. This delay is much greater than the residence time for positive ions or neutrals in the discharge region. Negative species could however remain trapped by the plasma electric potential for this duration [9] and act as precursors for particle growth.

Figures 3 and 6, using a simple model to interpret the experimental measurements, demonstrate that the negative ion density in the plasma is correlated with the onset rate and powder quantity above $1 \mathrm{kHz}$. Extrapolation to higher-mass negative ions in Figure 9 indicates that the macro-molecular anion densities would fall to near zero for a wide range of modulation frequencies below $1 \mathrm{kHz}$. This is because the plasma periods are then too short for the polymerisation chain to propagate from monosilicon hydride anions to highly-polymerised species before almost all of the low mass species are lost in the subsequent 'off' periods. Equilibrium between the diminished supply from preceding negative ions and the polymerisation of the polysilicon hydride anion itself results in a strongly-diminished density of the highlypolymerised anions (see Eq.(6) and Fig. 9). Above $1 \mathrm{kHz}$, the sheaths do not fully collapse and 
high-mass negative ions reach significant densities. At very low modulation frequencies [10], the polymerisation in a single plasma period propagates sufficiently far (to clusters containing perhaps 30 or 50 silicon atoms) to initiate particle growth. The onset rate in Figure 6 is a measure of the growth rate from precursors to detectable particles and consequently is an indication of the precursor density. Therefore the identification of negative ions as the precursors to particle formation $[12,42]$ is consistent with experimental observations of powder reduction in plasmas modulated at $\mathrm{kHz}$ frequencies $[8,10,12,16,17,19,42]$. Similar considerations also account for the observations in Fig. 8. We can now speculate the following stages of particle formation :

i) Dissociative electron attachment on silane creates the primary monosilicon hydride anions. Subsequent anion chemical reactions with silane, radicals, or positive ions yield highlypolymerised structured anionic clusters $[12,42]$ which are singly-charged and are trapped in the discharge by the rf electrode sheath potentials during continuous plasma operation. These anions are the precursors to cluster formation and ultimately lead to particles (see Ref. 43).

ii) After a certain cluster size is attained $[9,33,34]$, equilibration of electron and ion fluxes to its surface results in a multiple electronic surface charge and a sheath forms around the particle. This invisibly-small negatively charged nano-particle remains trapped in the plasma and continues to grow by physico-chemical accretion of neutrals and/or positive ions across the particle sheath.

iii) The nano-particles undergo an agglomeration phase and become large enough to be visible by light scattering (Fig. 4). Such processes are considered in the recent paper by Choi and Kushner [43]. Probably because the resultant micro-particles are composites of structured nanoparticles, they exhibit properties different from the bulk material such as a high refractive index $[44,45]$ and visible photoluminescence [24] (see Figs. 4(c) and 5). Particles continue to develop further by aggregation and processes analogous to film growth leading to particles with a-Si:H properties.

For plasma processing applications, restriction of the polymerisation to low mass anions could conceivably suppress particulate contamination and improve deposition efficiency. Alternatively to power modulation, this could perhaps be achieved by illuminating the plasma [32] with photodetaching radiation. 


\section{CONCLUSIONS}

The genesis and growth of particles in rf silane plasmas has been investigated by a combination of light scattering (for a self-consistent determination of particle size, number density and refractive index) and ion mass spectrometry. Time-resolved measurements of negative ion fluxes are interpreted in terms of a polymerisation scheme. Based on this, a simple model shows that anion cluster densities are depressed for the $\mathrm{kHz}$ range of power modulation frequencies corresponding to observed powder reduction. In addition, the observations that negative ions reach high masses and are the only species with a residence time on the scale of the powder growth time combine to show that negative ions are the precursors to particulate formation. Light scattering diagnostics demonstrate that particle evolution proceeds by an agglomeration phase and that the particles exhibit properties different from the bulk material, such as a high refractive index and visible photoluminescence.

\section{ACKNOWLEDGEMENTS}

We thank Dr. J. Perrin for valuable comments and suggestions. We also thank Dr. T. Stoto of Dept. IGA at EPFL for electron microscopy, and Hiden Analytical Limited. This work was funded by Swiss Federal Research Grant no. BBW.EG(91)3 (for BRITE/EURAM contract BE-4529-90) and by the Swiss Department of Energy Grant no. EF-REN(91)31. 


\section{REFERENCES}

[1] Selwyn G S, Singh J and Bennett R S 1989 J. Vac. Sci. Technol. A 72758

[2] Selwyn G S, McKillop J S, Haller K L and Wu J J 1990 J. Vac. Sci. Technol. A 81726

[3] Selwyn G S, Heidenreich J E and Haller K L 1991 J. Vac. Sci.Technol. A 92817

[4] Kushner M J 1988 J. Appl. Phys. 632532

[5] Veprek S, Schopper K, Ambacher O, Rieger W and Veprek-Heijman M G J to be published in J. Electrochem. Soc.

[6] Haller I 1980 Appl. Phys. Lett. 37282

[7] Perrin J, Lloret A, de Rosny G, and Schmitt J P M 1984 Int. J. Mass Spectrom. Ion Processes 57249

[8] Verdeyen J T, Beberman J and Overzet L 1990 J. Vac. Sci.Technol. A 81851

[9] Garscadden A, NATO ASI Series B: Physics Vol. 220. Nonequilibrium Processes in Partially Ionised Gases, edited by M. Capitelli and J. N. Bardsley, (Plenum, New York, 1990) 541; and

Garscadden A 1991 Proc. XXth Int. Conf. in Ionised Gases (Pisa), Invited Paper 147.

[10] Lloret A, Bertran E, Andújar J L, Canillas A, and Morenza J L 1991 J. Appl. Phys. 69632

[11] Boswell R W and Vender D 1991 IEEE Trans. on Pl. Sci. 19141

[12] Howling A A, Sansonnens L, Dorier J-L and Hollenstein Ch 1993 Lausanne Research Paper LRP $479 / 93$ (submitted to J. Appl. Phys., July 1993).

[13] Spears K G, Robinson T J and Roth R M 1986 IEEE Trans. Plasma Sci. 14 179; and Spears K G, Kampf R P, and Robinson T J 1988 J. Phys. Chem. 925297

[14] Bouchoule A, Plain A, Boufendi L, Blondeau J Ph and Laure C 1991 J. Appl. Phys. 70 1991

[15] Boufendi L, Plain A, Blondeau J Ph, Bouchoule A, Laure C and Toogood M 1992 Appl. Phys. Lett. 60169 
[16] Watanabe Y, Shiratani M, Kubo Y, Ogawa I and Ogi S 1988 Appl. Phys. Lett. 531263

[17] Watanabe Y, Shiratani M and Makino H 1990 Appl. Phys. Lett. 571616

[18] Watanabe Y, Shiratani M and Yamashita M 1992 Appl.Phys. Lett. 611510

[19] Howling A A, Hollenstein Ch and Paris P-J 1991 Appl. Phys. Lett. 591409

[20] Yoo W J and Steinbrüchel Ch 1992 J. Vac. Sci. Technol. A 101041

[21] Mie G 1908 Ann. Physik 25377

[22] Kerker M, The Scattering of Light and other electromagnetic radiation (Academic Press New York 1969)

[23] Jellum G M and Graves D B 1990 Appl. Phys Lett. 572077

[24] Dutta J, Dorier J-L, Hollenstein Ch, Howling A A, Sansonnens L, Nieswand C, Fasoli A and Stoto T 1993 Lausanne Research Paper LRP478/93 (submitted to Appl. Phys. Lett. July 93)

[25] Dorier J-L, Hollenstein Ch and Howling A A 1992 J. Vac. Sci. Technol. A 101048

[26] Bohren C F and Huffman D R 1983 Absorption and Scattering of Light by Small Particles (Wiley New York)

[27] Jellum G M, Daugherty J E and Graves D B 1991 J. Appl.Phys. 696923

[28] Kerker M, Scheiner P and Cooke D D 1978 J. Opt. Soc. Am. 68135

[29] Overzet L J, Lin Y and Luo L 1992 J. Appl. Phys. 725579

[30] Gottscho R A and Gaebe C E 1986 IEEE Trans. on Pl. Sci. PS-14 92

[31] Gottscho R A 1987 Phys. Rev. A 362233

[32] Howling A A, Sansonnens L, Dorier J-L, and Hollenstein Ch 1993 J. Phys. D: Appl. Phys. 261003 
[33] McCaughey M J and Kushner M J 1991 J. Appl. Phys. 696952

[34] Daugherty J E, Porteous R K, Kilgore M D and Graves D B 1992 J. Appl. Phys. 723934

[35] Mandich M L, Reents W D and Kolenbrander K D 1990 Pure \& Appl. Chem. 621653

[36] Weakliem H A, Estes R D and Longeway P A 1987 J. Vac. Sci. Technol. A 529

[37] Haaland P 1990 J. Chem. Phys. 934066

[38] Perrin J and Schmitt J 1992 Proc. 11th Eur. Photovoltaic Solar Energy Conf (Montreux) 80

[39] Blondeau J-Ph 1992 Thesis, Orleans University

[40] Bouchoule A and Boufendi L. to be published in Plasma Sources Science and Technol. Special Issue of the ESCAMPIG93 Conf.

[41] Heller W and Nakagaki M 1974 J. Chem. Phys. 613619

[42] Howling A A, Dorier J-L and Hollenstein Ch 1993 Appl. Phys. Lett. 621341

[43] Choi S J and Kushner M J 1993 J. Appl. Phys. 74853

[44] Brus L 1986 J. Phys. Chem. 902555

[45] Rossetti R, Ellison J L, Gibson J M and Brus L E 1984 J. Chem. Phys. 804464 


\section{FIGURE CAPTIONS}

FIG. 1 Schematic plan view of the diagnostic arrangement in the plane of a powder layer suspended in the plasma above the bottom electrode. The light source is a $10 \mathrm{~mW} \mathrm{He}-\mathrm{Ne}$ laser.

FIG. 2 Time-resolved fluxes for mono- to hexa-silicon hydride molecular anion groups measured at $1 \mathrm{kHz}$ power modulation frequency. Time is referenced to the beginning of a plasma period and the afterglow is from 500 to $1000 \mu \mathrm{s}$. The heavy line represents the following plasma duration. For each data point, the negative ions were counted with a $5 \mu$ s time window over $10^{\prime} 000$ modulation cycles. Pure silane at $0.1 \mathrm{mbar}$ and $4 \mathrm{~W}$ average power $\left(60 \mathrm{mWcm}^{-2}\right.$ during the plasma period) at $30 \mathrm{MHz}$ excitation frequency, electrode temperature $150^{\circ} \mathrm{C}$.

FIG. 3 Dependence of (a) positive and (b) negative ion signals on modulation frequency. Each data point is averaged over 300 cycles. The negative ion intensities are divided by the modulation frequency to give the loss counts per cycle. The plasma parameters are as in Fig. 2. The calculated frequency dependence of (c) the anion loss flux is shown for comparison, along with (d) the estimated anion densities in the plasma.

FIG. 4 Results from light scattering measurements during powder development in a cw plasma: (a) the solid lines are the measured scattered intensities (corrected for extinction) and the symbols represent the self-consistent fitted data; (b) the measured transmitted power fraction $W_{t} / W_{0}$ and polarisation degree $\mathrm{P}$; (c) the deduced particle radii, number density and refractive index values at $632.8 \mathrm{~nm}$. Pure silane at $0.1 \mathrm{mbar}$ and $30 \mathrm{MHz}$ with $5 \mathrm{~W} \mathrm{cw}$ power and $100{ }^{\circ} \mathrm{C}$ electrode temperature.

FIG. 5 Photoluminescence spectra from particles suspended in a pure silane plasma for different times during powder development after plasma ignition. Incident light at $488 \mathrm{~nm}$ wavelength. The spectra are corrected for the spectral response of the detection system and for the background plasma emission. Pure silane at $0.1 \mathrm{mbar}, 20 \mathrm{kHz}$ modulation at $5 \mathrm{~W}$ average rf power $(30 \mathrm{MHz})$ and $20^{\circ} \mathrm{C}$ temperature.

FIG. 6 The powder onset rate and volume of powder material suspended in the plasma as a function of square-wave power modulation frequency, compared with values in the continuous (CW) plasma. The powder material volume is estimated from $\left(N_{p} 4 \pi r^{3} / 3\right) \times$ (powder layer volume). Pure silane at $0.1 \mathrm{mbar}, 4 \mathrm{~W}$ average power at $30 \mathrm{MHz}$ and $100^{\circ} \mathrm{C}$.

FIG. 7 Simultaneous measurements of ion fluxes and powder development at $2 \mathrm{kHz}$ modulation frequency for (a) $50 \%$ duty cycle, and (b) $10 \%$ duty cycle. No powder is visible for the $10 \%$ duty cycle. Plasma parameters as for Fig. 6 . 
FIG. 8 Powder onset rate, steady-state scattered intensity and ion fluxes for different duty cycles at $2 \mathrm{kHz}$ modulation frequency. Pure silane at $0.1 \mathrm{mbar}, 4 \mathrm{~W}$ average power at $30 \mathrm{MHz}$ and $80^{\circ} \mathrm{C}$.

FIG. 9 Estimation of time-averaged anion densities, as a function of modulation frequency, extrapolated to anions containing 25 silicon atoms (polymerisation rates assumed independent of size for anions of 6 or more silicon atoms). Insets show the time-varying density during a modulation cycle for three frequencies : at $100 \mathrm{~Hz}$, the polymerisation chain has insufficient time to propagate to the highest masses; at $1 \mathrm{kHz}$, the elementary anions are lost before significant polymerisation can occur; at $20 \mathrm{kHz}$, all anions are trapped and reach high densities. 


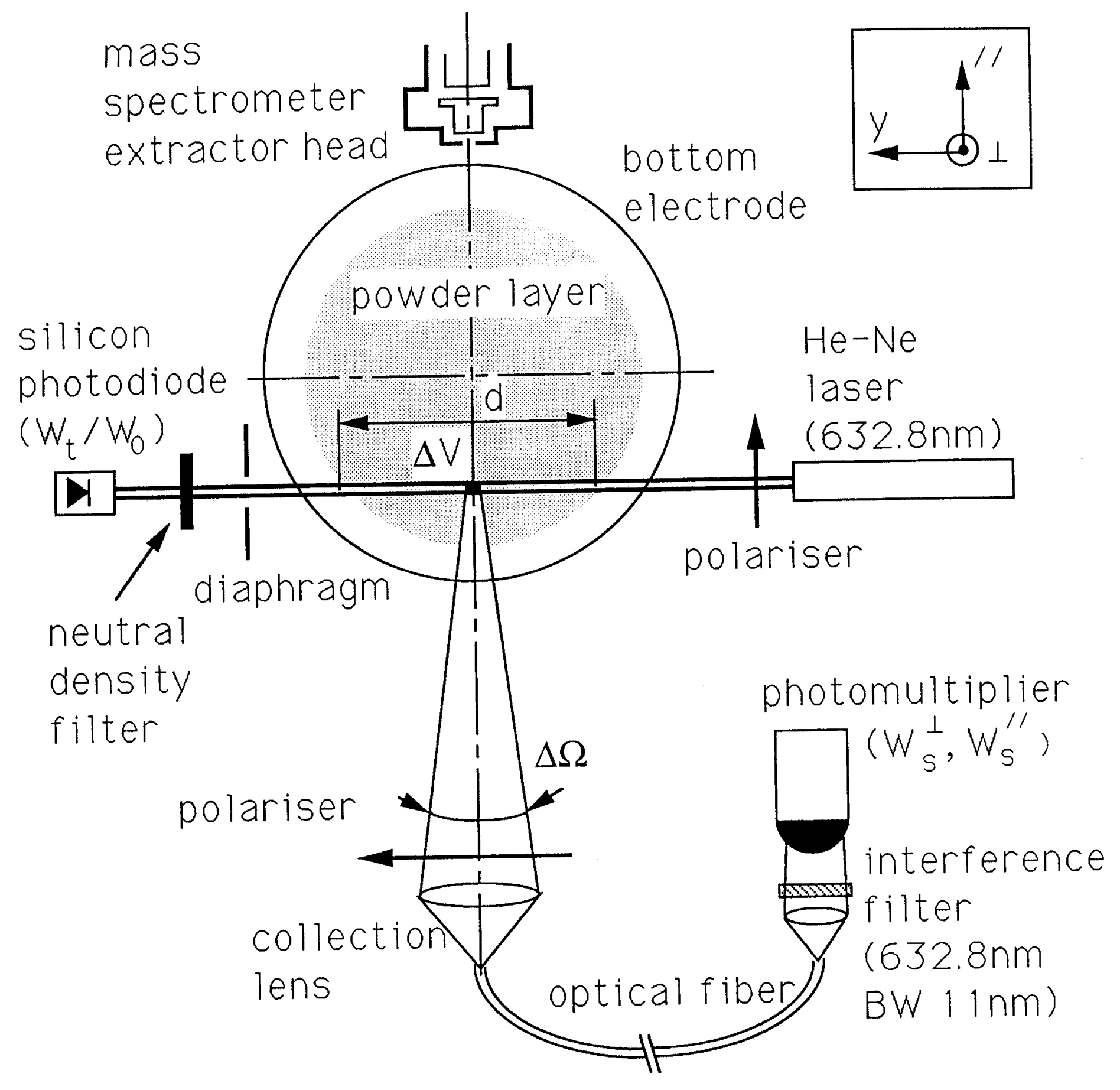

Fig. 1 


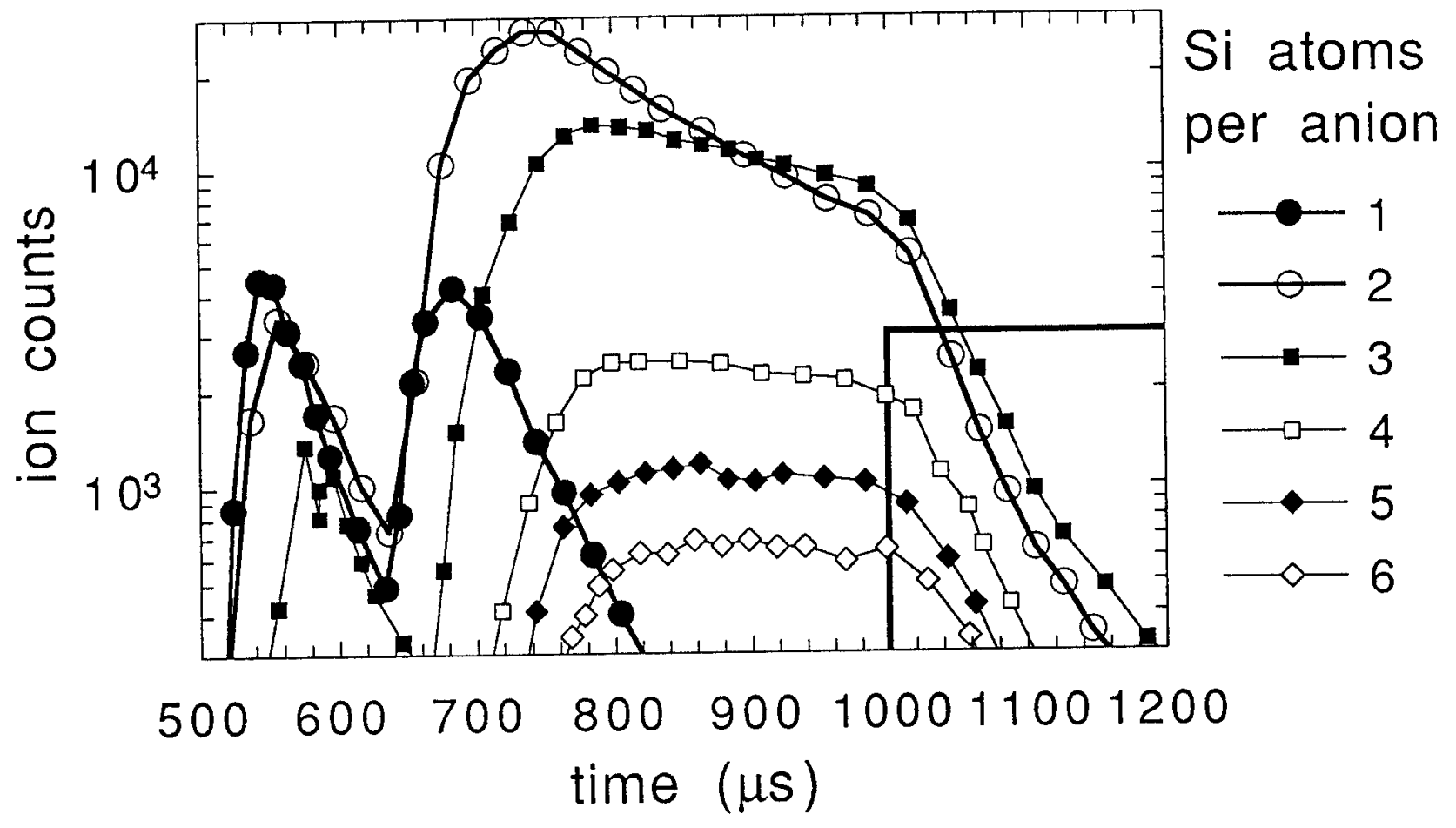

Fig. 2 


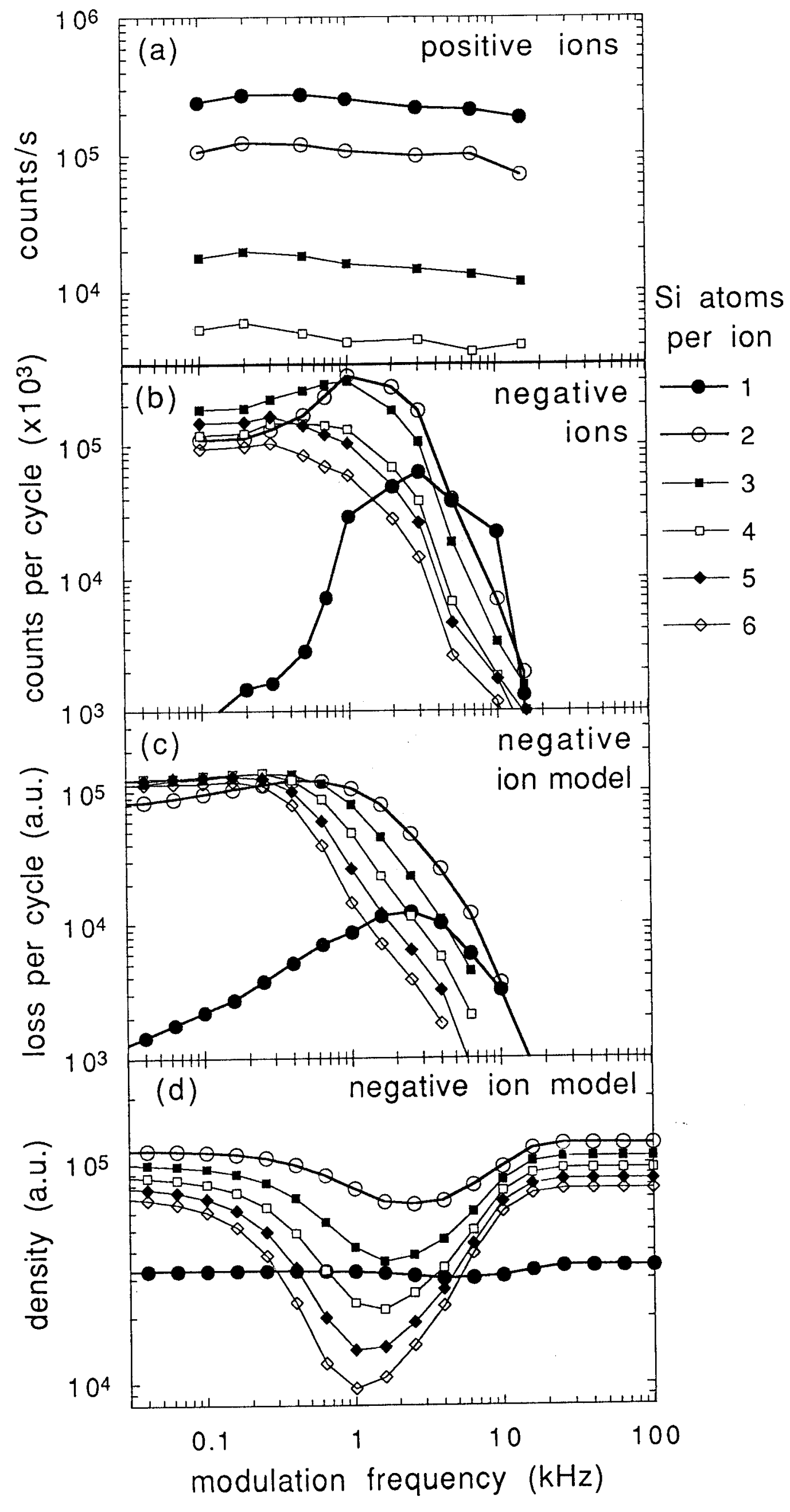

Fig. 3 


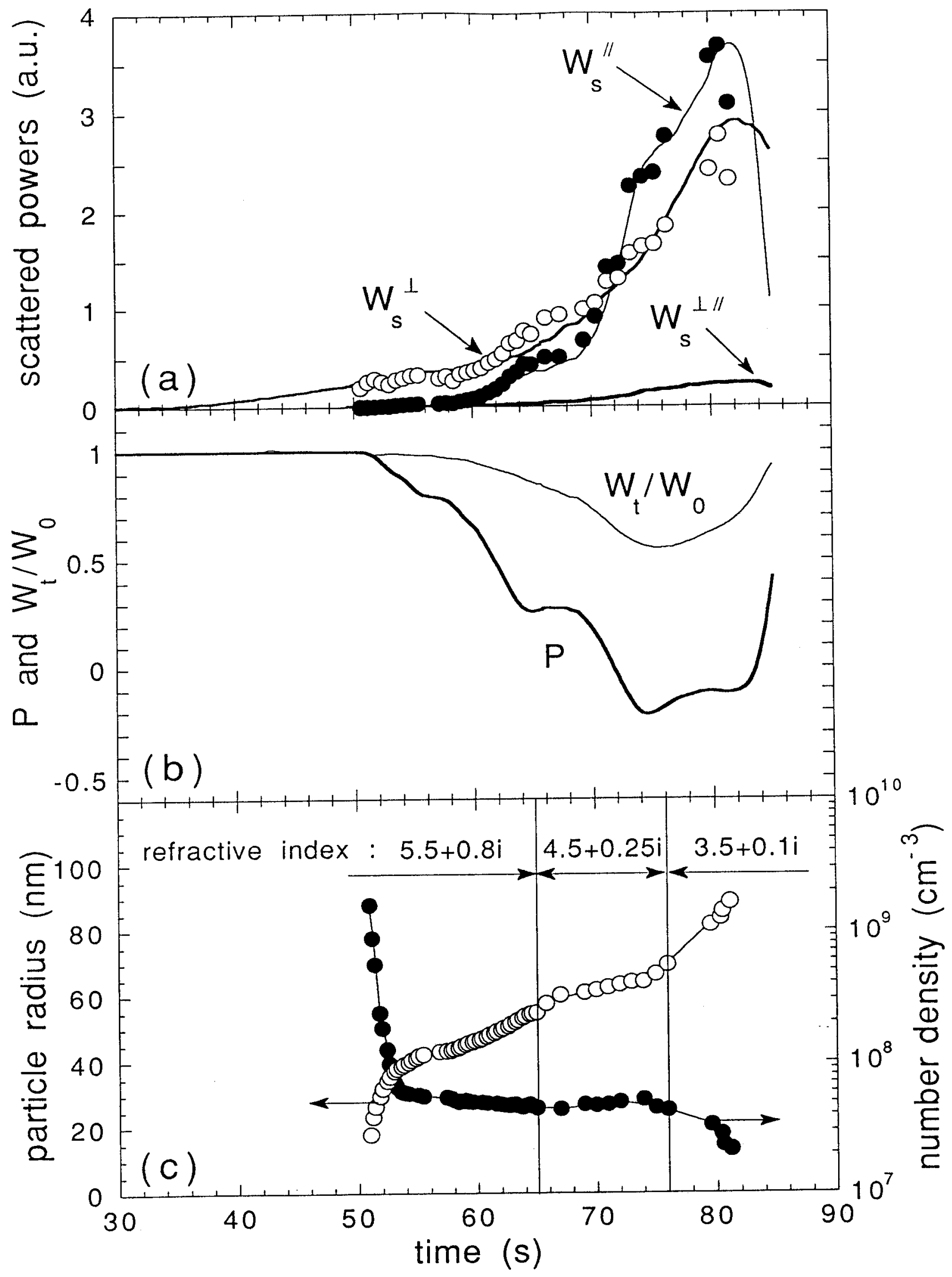

Fig. 4 


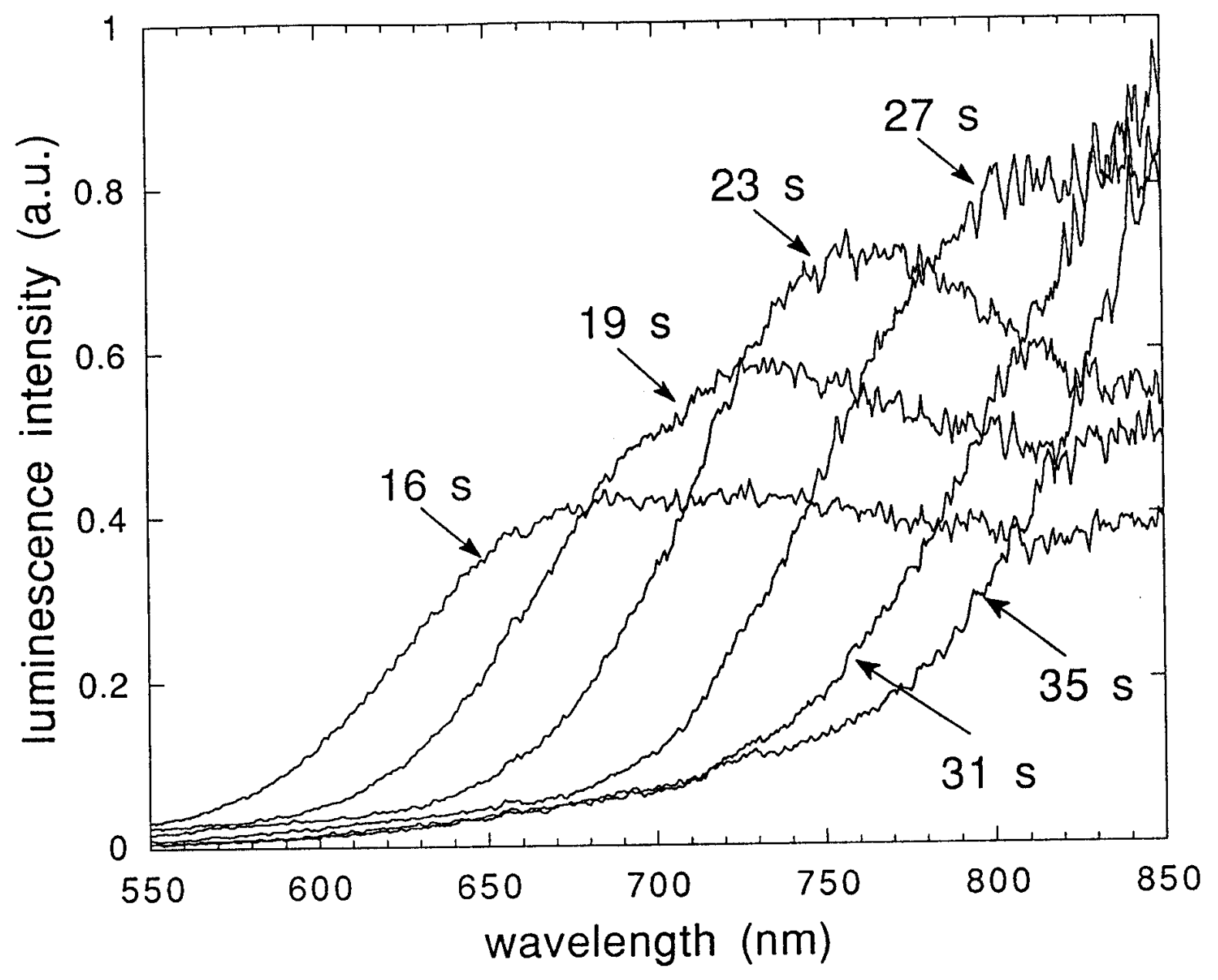

Fig. 5 


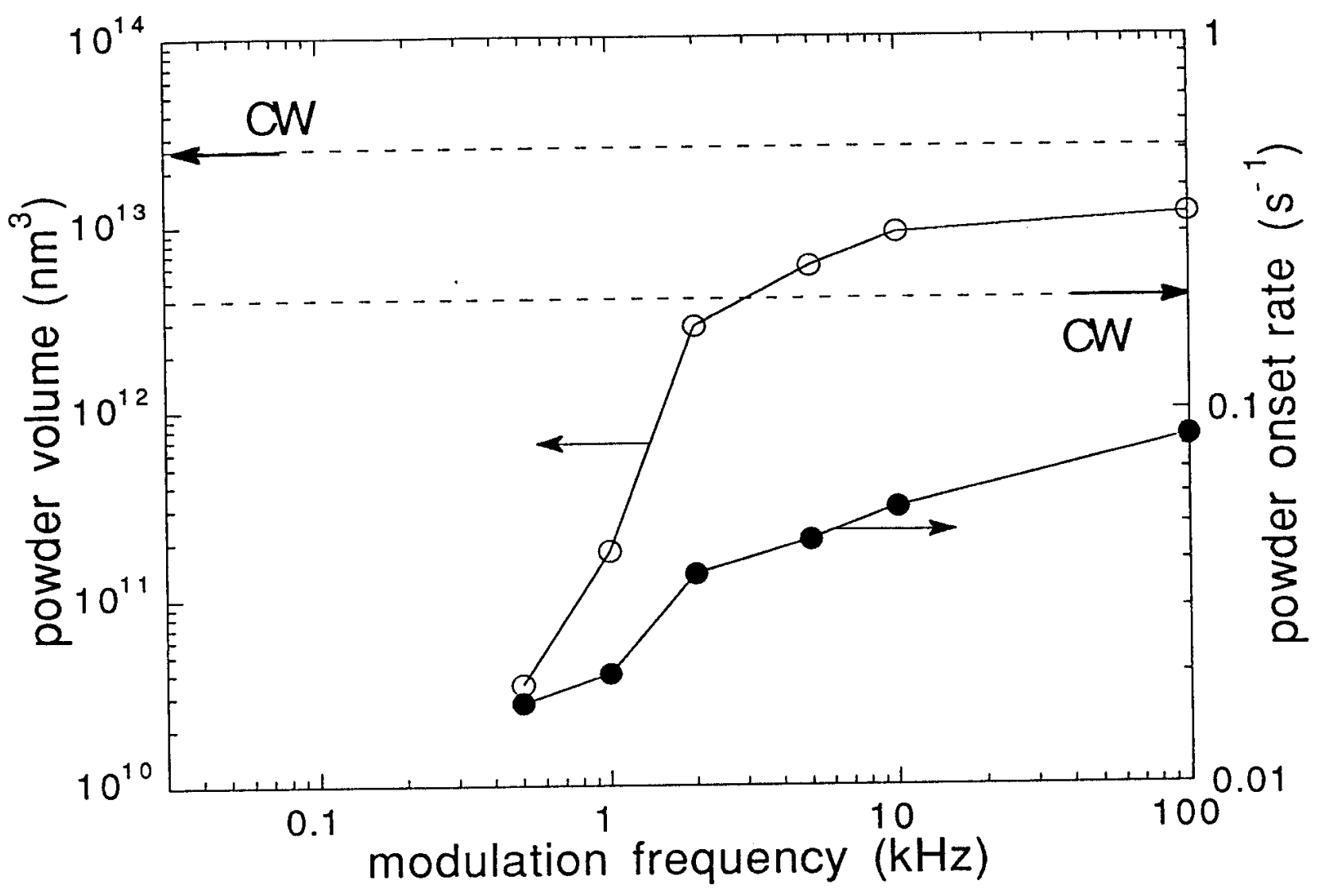

Fig. 6 


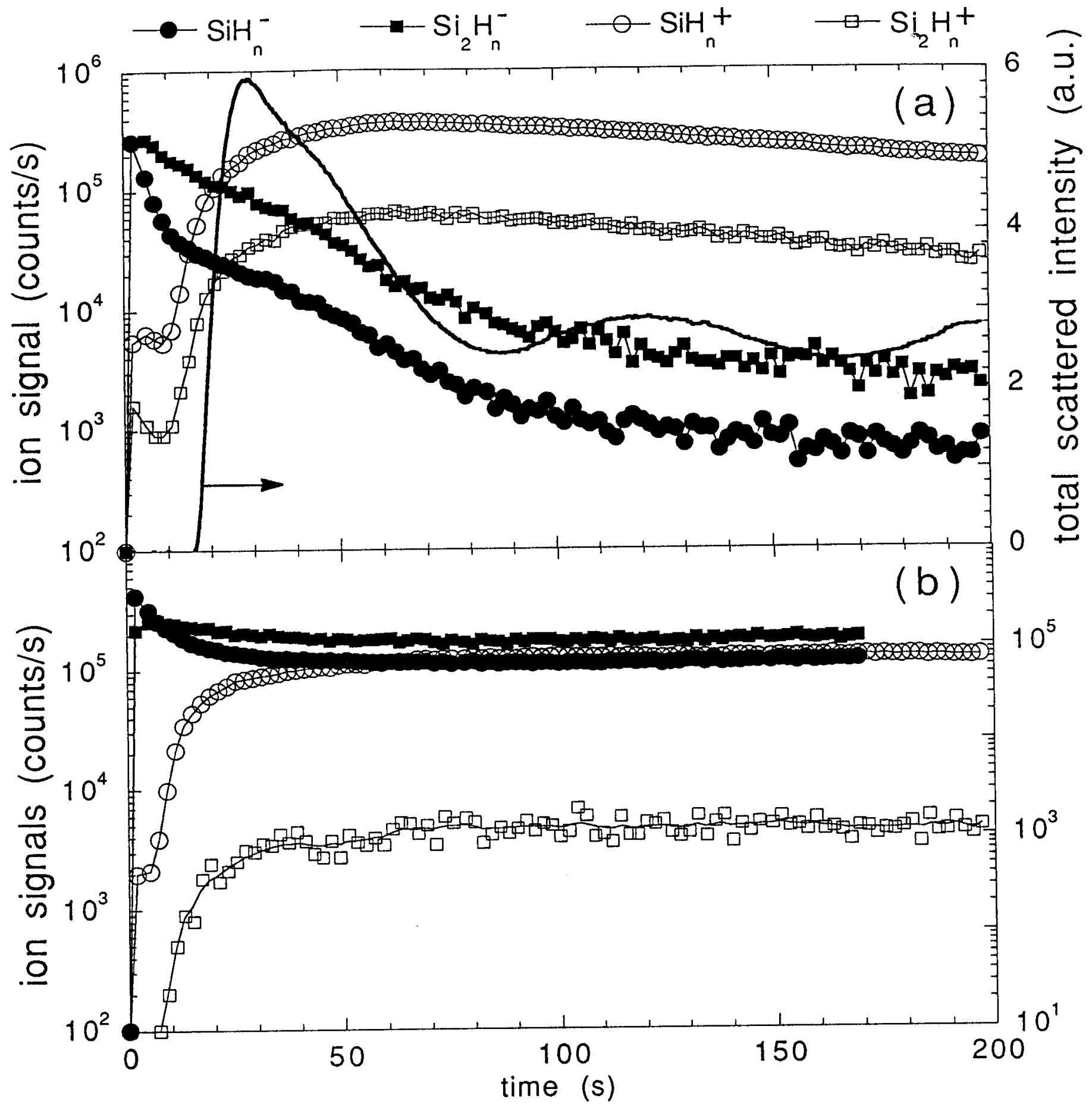

Fig. 7 


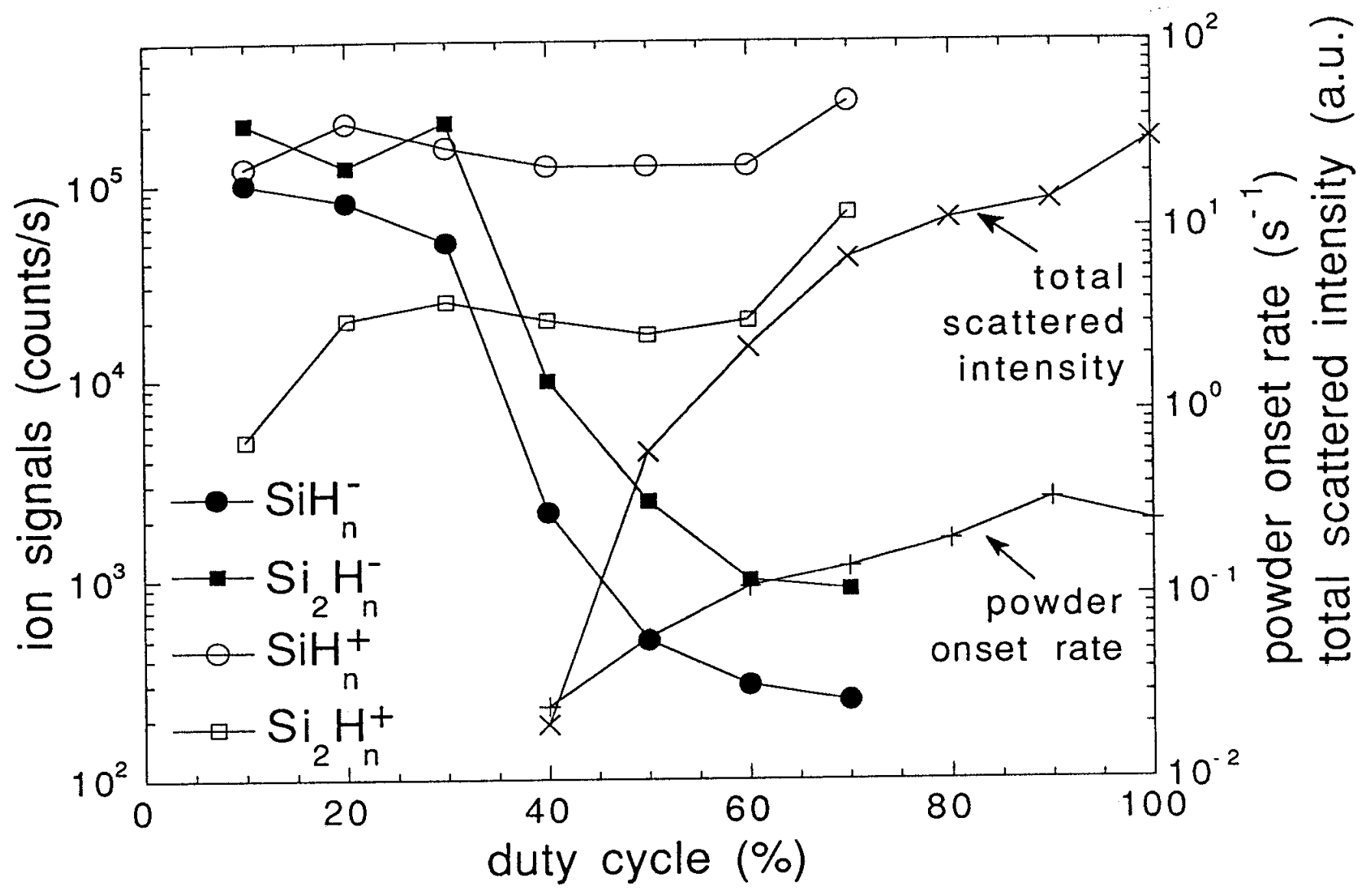

Fig. 8 

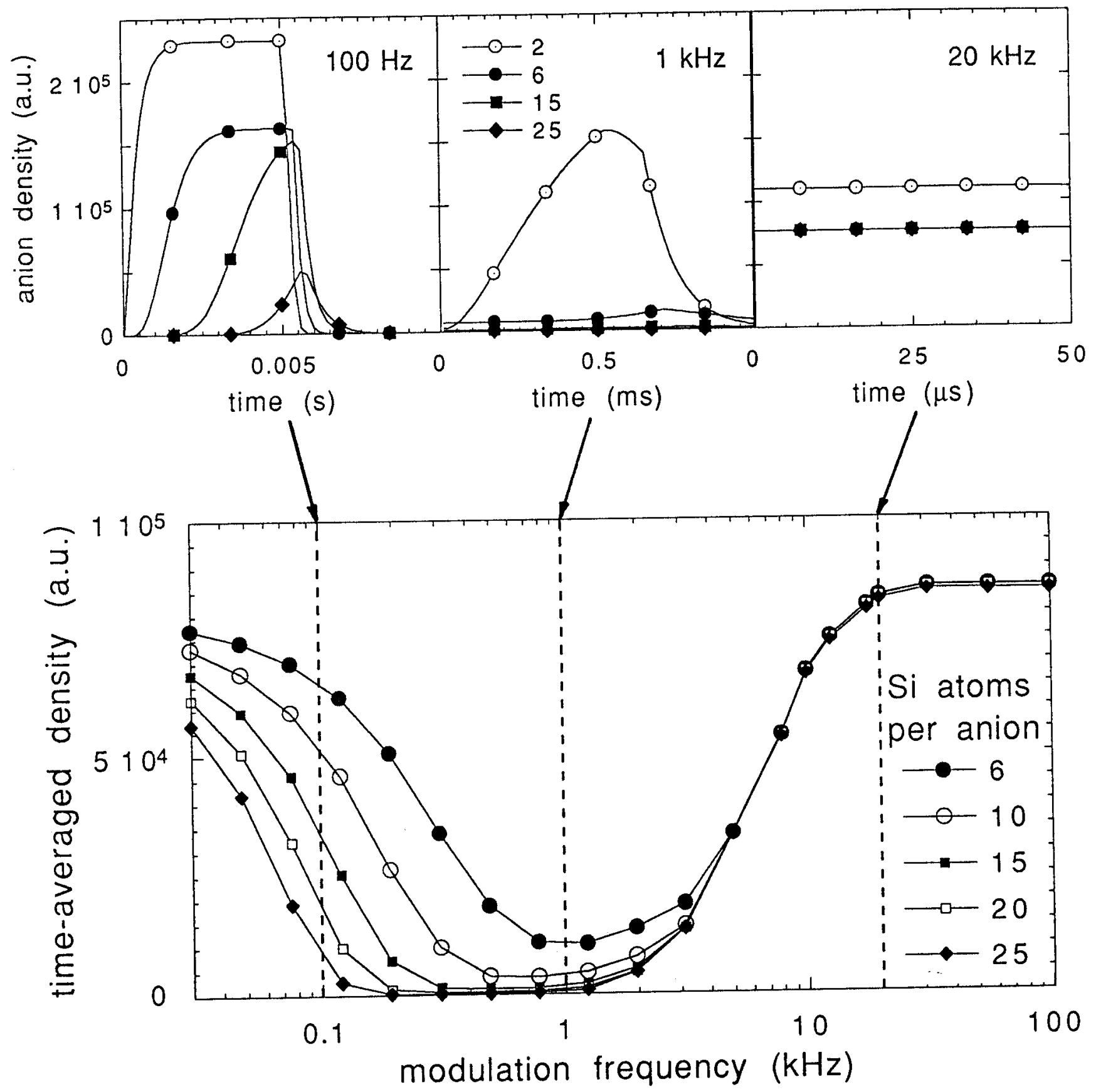

Fig. 9 